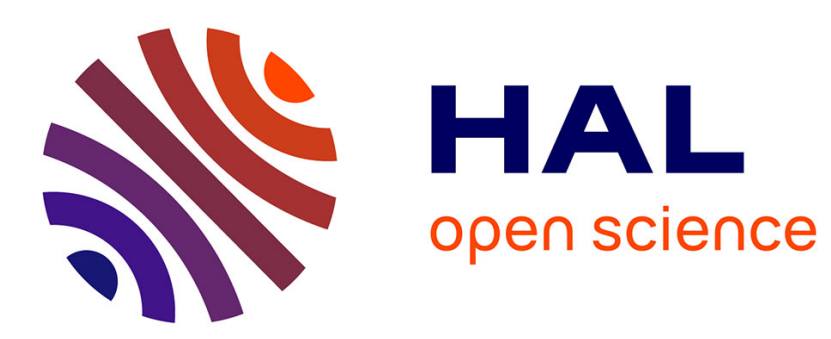

\title{
e-COMM, un éditeur pour spécifier l'interaction multimodale et multiutilisateur
}

Frédéric Jourde, Yann Laurillau, Laurence Nigay

\section{To cite this version:}

Frédéric Jourde, Yann Laurillau, Laurence Nigay. e-COMM, un éditeur pour spécifier l'interaction multimodale et multiutilisateur. Actes de la 22ème Conférence francophone sur l'Interaction HommeMachine (IHM'2010, Luxembourg, Septembre 2010), 2010, Unknown, Luxembourg. pp.225-228, 10.1145/1941007.1941048. hal-00953325

\section{HAL Id: hal-00953325 \\ https://hal.inria.fr/hal-00953325}

Submitted on 28 Feb 2014

HAL is a multi-disciplinary open access archive for the deposit and dissemination of scientific research documents, whether they are published or not. The documents may come from teaching and research institutions in France or abroad, or from public or private research centers.
L'archive ouverte pluridisciplinaire HAL, est destinée au dépôt et à la diffusion de documents scientifiques de niveau recherche, publiés ou non, émanant des établissements d'enseignement et de recherche français ou étrangers, des laboratoires publics ou privés. 


\title{
e-COMM, un éditeur pour spécifier l'interaction multimodale et multiutilisateur
}

\author{
Frédéric Jourde, Yann Laurillau, Laurence Nigay \\ Université de Grenoble, Laboratoire LIG \\ Domaine Universitaire \\ 38000 Grenoble, France \\ \{frederic.jourde, yann.laurillau, laurence.nigay\}@imag.fr
}

\begin{abstract}
RESUME
Cet article présente l'éditeur e-COMM pour la spécification de systèmes interactifs multimodaux et multiutilisateurs à l'aide de la notation COMM. La majorité des notations dédiées à la conception des collecticiels offrent des moyens limités pour décrire l'interaction multimodale. Ainsi la notation COMM comble ce manque en introduisant de nouveaux concepts (tâche modale et rôle interactif) pour lier ces deux aspects : l'interaction multimodale et multiutilisateur. L'éditeur e-COMM vise alors deux objectifs :founir un outil facilement accessible et aider le concepteur à se concentrer sur la tâche d'édition en reposant autant que possible sur la manipulation directe.
\end{abstract}

MOTS CLES : Collecticiels, interaction multimodale, notation de spécification.

\begin{abstract}
Addressing this issue of lack of design tools for multiuser multimodal systems, we present the COMM (Collaborative and MultiModal) notation and its on-line editor for specifying multi-user multimodal interactive systems. Extending the CTT notation, the salient features of the COMM notation include the concepts of interactive role and modal task. The e-COMM graphical user interface emphasizes direct manipulation of the COMM concepts in order to avoid forms, menus or toolbars as much as possible. Such UI should encourage focus on the specification under development.
\end{abstract}

CATEGORIES AND SUBJECT DESCRIPTORS: H.5.3. Group and Organization Interfaces: Computersupported supported cooperative work. H.5.2. User Interfaces: Input devices and strategies. D.2.2. Design Tools and Techniques: User interfaces.

GENERAL TERMS: Human Factors; Design; Algorithms.

KEYWORDS: Groupware, multimodal interaction, specification notation.

IHM'10 (C) ACM, 2010. This is the author's version of the work. It is posted here by permission of ACM for your personal use. Not for redistribution. The definitive version was published in Actes de la 22ème Conférence Francophone sur l'Interaction Homme-Machine, IHM'10.

\section{INTRODUCTION}

Dans cet article, nous présentons l'éditeur e-COMM disponible sous la forme d'une application en ligne via un navigateur web (iihm.imag.fr/demo/editeur). Cet éditeur repose sur la notation COMM [4] pour permettre et faciliter la spécification d'un système interactif multimodal et multiutilisateur. Un système interactif est multimodal lorsqu'un utilisateur peut interagir avec le système par l'usage simultané de plusieurs modalités d'interaction (en entrée ou en sortie). Aussi, nous observons que de plus en plus de collecticiels sont multimodaux tels les systèmes développés et décrits dans $[1,5]$.

De plus, il y a un intérêt croissant au sein de la communauté du Travail Collaboratif Assisté par Ordinateur (TCAO) pour les systèmes interactifs multimodaux et multiutilisateurs construits autour des surfaces interactives $[9,11,13]$. Toutefois, le développement de tels systèmes reste ad hoc. En particulier, leur conception soulève de nombreuses questions sur la disponibilité des différentes modalités et de leur combinaison en fonction des rôles endossés.

Pour aborder la spécification des systèmes interactifs multimodaux et multiutilisateurs, nous avons adopté une approche s'appuyant sur les notations à base d'arbre de tâches. Nous avons étudié de nombreuses notations existantes [3] comme CTT [8], GTA [14], MABTA [6], CUA [10]. Nous avons mis en évidence des lacunes pour décrire la nature multimodale de l'interaction multiutilisateur, ce qui a motivé l'élaboration de la notation COMM. Celle-ci se présente comme une évolution de la notation CTT et introduit de nouveaux concepts : le rôle interactif et la tâche modale. Le développement de l'éditeur eCOMM, adossé à la notation COMM, s'est révélé être une évidence et indispensable pour faciliter l'usage de la notation. En effet, comme le souligne Van der Veer [15] et Molina [7], une notation est réellement utilisable si le concepteur dispose d'outils facilitant son usage. Ceci a donc motivé la réalisation de l'éditeur.

Ce travail s'inscrit dans le cadre du projet PEA FH-PA (Facteurs Humains - Partage d'Autorité) piloté par la DGA, Bertin Technologies, EADS, Sagem et PY Automation. Le domaine d'application est le poste de commande de drones. La notation COMM et l'éditeur eCOMM sont utilisés dans le cadre de ce projet pour spécifier une nouvelle génération de postes de commande.

Dans la suite, nous présentons les principaux éléments de la notation COMM puis les principales fonctionnalités de 
l'éditeur e-COMM et les principes qui ont guidé sa conception.

\section{NOTATION COMM}

La notation COMM se présente comme une extension de CTT par l'introduction de deux concepts, le rôle interactif et la tâche modale, et par la spécialisation des opérateurs temporels, en s'appuyant sur les cinq relations de Allen [16], afin de décrire finement l'articulation entre les tâches concrètes :

- Un rôle interactif est associé à une combinaison de modalités. Cette notion diffère de la notion usuelle de rôle telle qu'employée dans la littérature du domaine du TCAO. Cette dernière caractérise très souvent un rôle métier, c'est-à-dire le rôle d'un acteur au sein d'une organisation. Il reflète donc une organisation logique de l'activité de groupe. A contrario, le rôle interactif permet de mettre en évidence dans quelle mesure une activité de groupe est influencée par des contraintes liées à l'interaction. Ainsi, ces deux notions de rôles permettent de rendre explicite les contraintes liées à l'activité de groupe et les contraintes liées à l'interaction concrète.

- Une tâche modale, représentée à la Figure 1, est un type de tâche complémentaire pour décrire une interaction concrète élémentaire : ce type de tâche permet de décrire une action utilisateur atomique réalisée avec un périphérique donné.

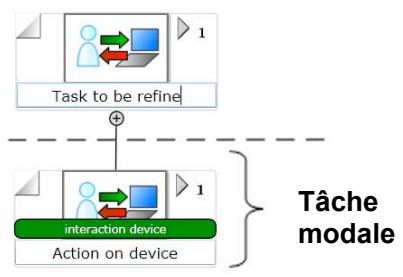

Figure 1 : Tâche modale.

- Les relations de Allen, représentées à la Figure 2, sont employées pour décrire plus finement les relations temporelles entre les tâches abstraites au niveau concret. Ces dernières se limitent à du séquentiel ou du parallélisme.

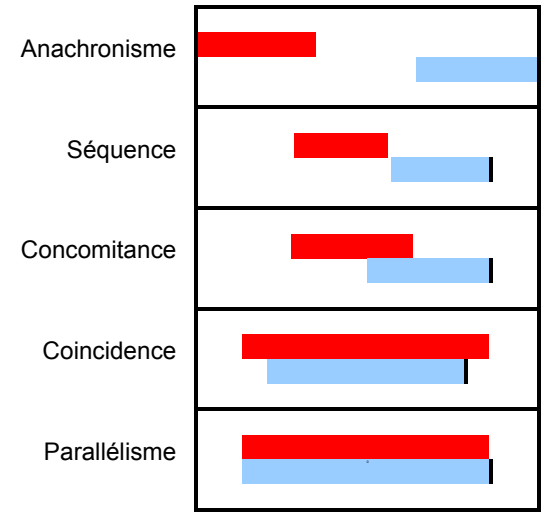

Figure 2 : Représentation schématique des relations temporelles de Allen.

\section{Principaux symboles de la notation}

Nous proposons plusieurs pictogrammes pour décrire les tâches multiutilisateurs et les tâches individuelles. Comme le montre la Figure 3, il existe trois types de tâches individuelles (symboles avec un personnage) : tâche action (sans retour d'information), tâche interaction (avec retour), tâche mentale (interne). Ces trois types sont déclinés pour les tâches de groupe (symboles avec trois personnages). En particulier, la tâche mentale se décline en une tâche de coordination comme une prise de décision au sein du groupe sans l'intervention du système. A ces six types, s'ajoutent deux autres types : une tâche système qui représente une action interne au système et une tâche de présentation (retour d'information). Cette dernière peut être déclenchée explicitement par l'utilisateur suite à une tâche action ou à l'initiative du système. A ces symboles représentant les différents types de tâches s'ajoutent des pictogrammes complémentaires permettant de décorer chaque tâche. Deux pictogrammes (voir Figure 3) permettent d'indiquer si une tâche relève de l'interaction abstraite (symbole de nuage) ou de l'interaction concrète (symbole carré). Lorsqu'il s'agit d'une tâche de groupe, un symbole représentant un masque accompagné d'une légende indique quels sont les rôles associés à la tâche. Le dernier symbole indique qu'il y a un nombre fixe d'utilisateurs pouvant réaliser une tâche simultanément. Dans le cas d'une tâche concrète et collaborative, ce symbole est accompagné d'une légende précisant quel est le rôle interactif impliqué pour la réalisation de cette tâche. Cette légende sous-entend que ce rôle interactif sera endossé par l'un des rôles métiers défini au niveau de la tâche abstraite. Par contre, l'association d'un rôle interactif à un rôle métier se décide au moment de l'exécution de la tâche. En effet, par définition, une tâche collaborative ne prévoit pas une répartition a priori des tâches contrairement à une tâche coopérative [2].

\begin{tabular}{|c|c|c|c|}
\hline \multicolumn{4}{|c|}{ Types de tâches } \\
\hline$\Rightarrow$ & $\begin{array}{c}\text { Action } \\
\text { utilisateur }\end{array}$ & & $\begin{array}{c}\text { Action } \\
\text { de groupe }\end{array}$ \\
\hline$\Rightarrow$ & $\begin{array}{l}\text { Interaction } \\
\text { utilisateur }\end{array}$ & & $\begin{array}{l}\text { Interaction } \\
\text { de groupe }\end{array}$ \\
\hline & Tâche mentale & & $\begin{array}{c}\text { Tâche } \\
\text { de coordination }\end{array}$ \\
\hline & Tâche système & $\sum$ & $\begin{array}{c}\text { Tâche } \\
\text { de présentation }\end{array}$ \\
\hline \multicolumn{4}{|c|}{ Décorations } \\
\hline & Tâche abstraite & & Tâche concrète \\
\hline & Rôle métier & 8 & $\begin{array}{l}\text { Nombre de rôles } \\
\text { impliqués }\end{array}$ \\
\hline
\end{tabular}

Figure 3 : Principaux symboles de la notation COMM.

\section{EDITEUR E-COMM}

L'éditeur e-COMM est développé sous la forme d'une application en ligne (iihm.imag.fr/demo/editeur). Celuici repose sur la technologie SilverLight de Microsoft et est programmé en C\#. Cette application (voir Figure 4) est accessible via n'importe quel navigateur web et ne nécessite aucune installation outre le plug-in SilverLight. L'interaction a été pensée pour favoriser autant que possible la manipulation directe et pour limiter autant que possible la multiplication des fenêtres pop-up. La principale fenêtre pop-up disponible a été pensée pour limiter le nombre de champs du formulaire (Figure 5). Aussi, l'espace d'affichage disponible est principalement un espace d'édition dédié à la construction d'arbres. 


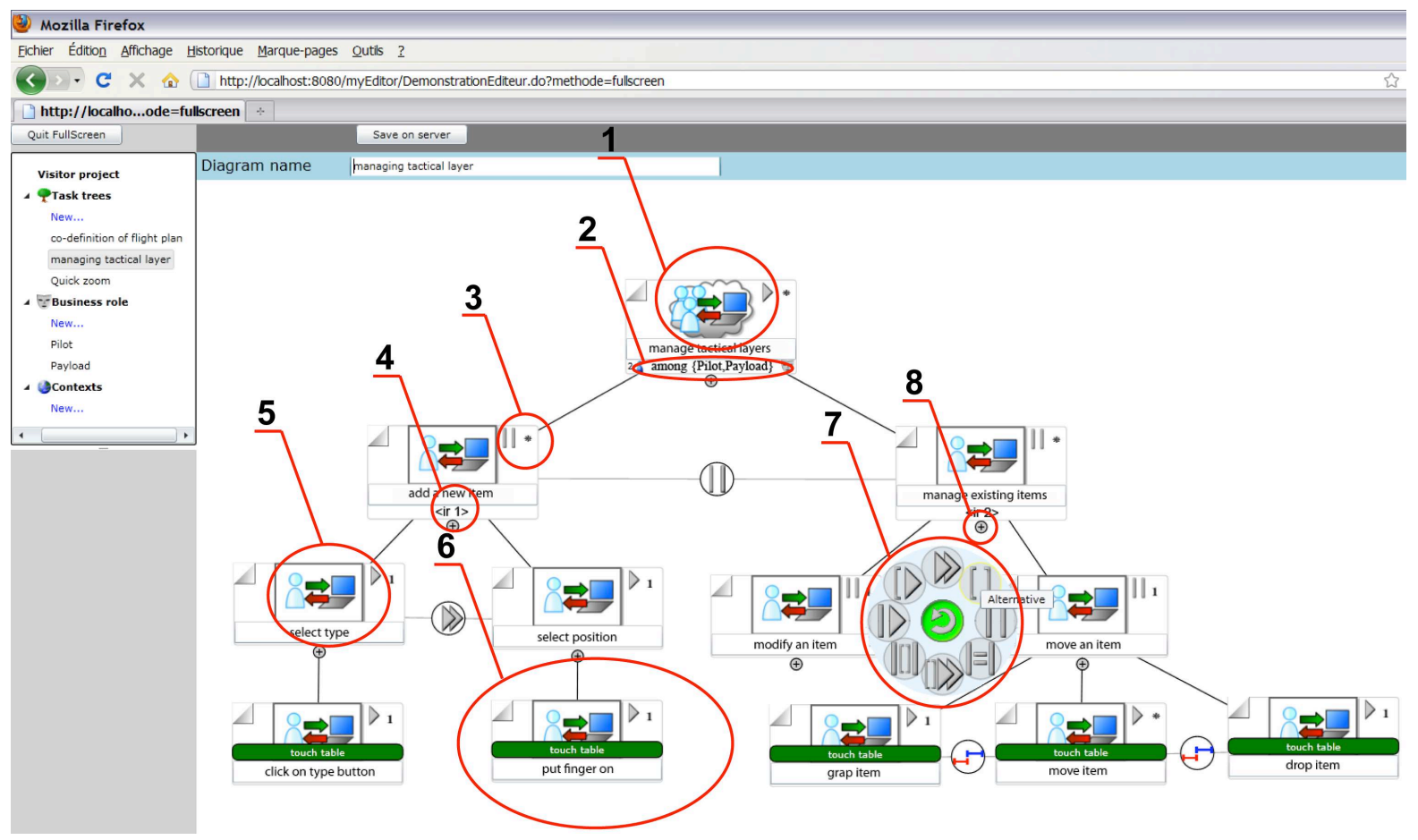

Figure 4 : Copie d'écran de l'éditeur e-COMM.

Les différents espaces de travail sont accessibles par une barre verticale située sur le côté gauche de l'interface. A cela s'ajoute une barre de commandes réduite à quelques boutons dans la partie supérieure de l'interface. Lors de la création d'un nouvel arbre, l'éditeur s'ouvre avec une seule tâche. L'ajout de tâches filles est réalisable en cliquant sur le symbole (+) (Figure 4-8) situé en dessous de chaque tâche. Pour attribuer un nom à une tâche, il suffit de cliquer dans la zone inférieure du rectangle associé et de réaliser une saisie au clavier. Si une tâche se révèle être mal placée, il suffit de la déplacer (technique glisser/déposer) vers une autre tâche. L'éditeur fait alors apparaître tous les points d'ancrage possibles à la manière d'un aimant. Pour la suppression, il suffit de sélectionner une tâche et d'activer la suppression au clavier. Bien sûr, l'éditeur supporte la sélection et le déplacement d'une grappe de tâches, le zoom avant et arrière, et le déplacement de la zone de travail. Dès que l'on ajoute une nouvelle tâche fille, celle-ci est reliée par un opérateur inconnu. Pour choisir un des opérateurs CTT, hérités de LOTOS, il suffit de cliquer sur l'opérateur. Une palette circulaire apparaît (Figure 4-7). Un second clic au centre de la palette fait apparaître la palette circulaire d'opérateurs temporels basés sur les relations de Allen.

Une partie des symboles de la notation COMM se retrouvent au sein de la Figure 4 (annotations 1, 2, 3, 4, 5 et 6). Ces derniers sont modifiables au travers de l'unique formulaire dédié à la décoration des tâches. Comme le montre la Figure 5, il est possible (i) de modifier le nom de la tâche, ce qui est déjà réalisable directement comme évoqué précédemment, (ii) de choisir un type de tâche parmi la liste décrite dans la partie précédente (le symbole associé est alors mis à jour), (iii) de définir le nombre d'itérations à l'aide d'un opérateur unaire et de préciser si ces itérations sont réalisables en séquence ou en parallèle (le symbole correspondant ap- paraît alors dans la représentation de la tâche (Figure 43)), (iv) de préciser s'il s'agit d'une tâche abstraite (Figure 4-1) ou concrète (Figure 4-5), (v) d'indiquer quels sont les rôles métiers (Figure 4-2) ou interactifs impliqués (Figure 4-4), (vi) de définir quel dispositif est employé pour réaliser la tâche lorsqu'il s'agit d'une tâche concrète et modale (Figure 4-6), (vii) de spécifier des pré- et post-conditions (Figure 5).

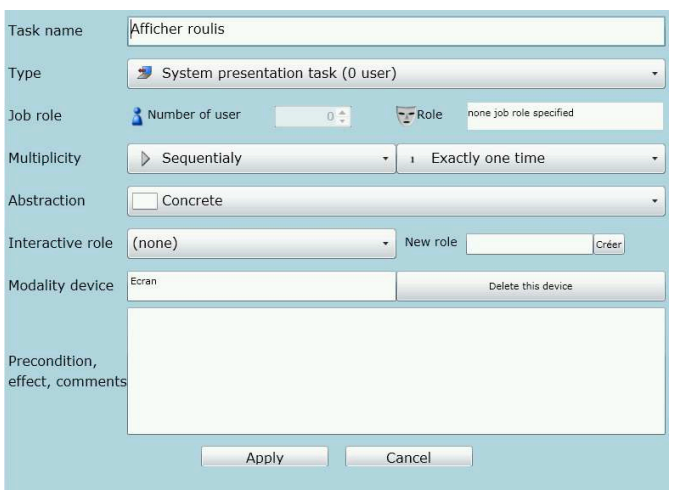

Figure 5 : Formulaire pour la décoration des tâches.

Afin de faciliter la gestion des créations, les arbres sont sauvegardés sur le serveur. Toutefois, l'éditeur propose deux modes d'exportation. Le premier permet de récupérer les arbres sous la forme d'une image PNG en haute résolution, ce qui en facilite la manipulation pour une insertion dans un document. Le second mode permet une exportation au format XML si on souhaite intégrer la description avec d'autres outils. Enfin, dans la barre verticale située dans la partie gauche de l'interface, il est possible de gérer plusieurs arbres pour un même projet, de créer plusieurs rôles définis par un profil, ceux-ci étant utilisés dans le formulaire dédié à la décoration de la tâche. 


\section{CONCLUSION}

L'éditeur e-COMM est pleinement fonctionnel et est employé dans le cadre du projet PEA FH-PA piloté par la DGA et des partenaires industriels (Bertin, EADS, Sagem et PY Automation). Avec cet éditeur, nous avons été en mesure de spécifier la première version du prototype d'interface de poste de commande de drone. Les arbres obtenus ont une profondeur de 12 niveaux de tâches pour plus de 150 tâches feuilles. Le soin apporté à l'interaction lors de la conception de l'éditeur, en s'appuyant autant que possible sur la manipulation directe, se ressent à l'usage. Cet aspect a grandement facilité le travail de spécification pour ce projet. A cet usage, s'ajoute un projet avec 16 étudiants du Master 2 de l'Université Joseph Fourier dans le cadre du cours sur les collecticiels. Ce travail a pour but de mettre à l'épreuve la notation COMM et l'éditeur e-COMM afin de fournir des éléments d'évaluation de la notation et des retours d'usage de l'éditeur. Ce projet n'est pas encore terminé mais les premiers retours sur l'usage de l'éditeur sont très positifs. Enfin, bien que le développement de l'éditeur soit bien abouti, celui-ci est poursuivi afin de permettre l'ajout de nouvelles fonctionnalités comme le copier/coller d'une grappe de tâches, l'import d'une description XML d'un arbre, de réduire un sous-ensemble pour gagner en lisibilité et espace d'affichage.

\section{BIBLIOGRAPHIE}

1. Brown, B., MacColl, I., Chalmers, M., Galani, A., Randell, C., and Steed, A., Lessons from the lighthouse: collaboration in a shared mixed reality system, in Proceedings of CHI'03 (Ft. Lauderdale FL, April 2003), ACM Press, 577-584.

2. Dillenbourg, P., Baker, M., Blaye, A., and O'Malley, C. The evolution of research on collaborative learning. Learning in Humans and Machine: Towards an interdisciplinary learning science, 1996, 189-211, Elsevier.

3. Jourde, F., Laurillau, Y., Morán, A., and Nigay, L., Towards Specifying Multimodal Collaborative User Interfaces: A Comparison of Collaboration Notations, in Proceedings of DSV-IS'08 (Kingston, Canana, July 2008), Springer, 281-286.

4. Jourde, F., Laurillau, Y., and Nigay, L., COMM Notation for Specifying Collaborative and MultiModal Interactive Systems, in Proceedings of EICS'10 (Berlin, Germany, June 2010), ACM Press, à paraître.

5. Kraut, R.E., Fussel, S.R., and Siegel, J. Visual Information as a Conversational Resource in Collaborative Physical Tasks. Human-Computer Interaction Journal, 2003, 18(1), 13-49, Lawrence Erlbaum.
6. Lim, Y.K., Task models for groupware and multitasking: Multiple aspect based task analysis (MABTA) for user requirements gathering in highly-contextualized interactive system design, in Proceedings of TAMODIA'04 (Prague, Czech Republic, 2004), ACM Press, 7-15.

7. Molina, A.I., Redondo, M.A., and Ortega, M. A Review of Notations for Conceptual Modeling of Groupware Systems. New Trends on HumanComputer Interaction, 2009, 75-86, Springer.

8. Mori, G., Paternó, F.,Santoro, C., CTTE : Support for Developing and Analyzing Task Models for Interactive System Design. TOSE Journal, 2002, 28(8), 797-813, IEEE Press.

9. Pauchet, A., Colfedy, F., Lefebvre, L., Picard, S., Bouguet, A., Perron, L., Guerin, J., Corvaisier, D., and Collobert, M., Mutual Awareness in Collocated and Distant Collaborative Tasks Using Shared Interfaces, in Proceedings of INTERACT'07 (Rio de Janeiro, Brazil, September 2007), Springer, 59-73.

10. Pinelle, D., Gutwin, C., and Greenberg, S. Task Analysis for Groupware Usability Evaluation: Modeling Shared-Workspace Tasks with the Mechanics of Collaboration. Transactions on Computer-Human Interaction (TOCHI), 2003, 10(4), 281-311, ACM Press.

11. Tang, A., Tory, M., Po, B., Neumann, P., and Carpendale, S, Collaborative Coupling over TableTop Displays, in Proceedings of ACM CHI'06 (Montréal, Québec, Canada, April 2006), ACM Press, 1181-1190.

12. Treatteberg, H. Model-based User Interface Design. PhD Thesis, 2002, Norwegian University of Science and Technology.

13. Tse, E., Shen, C., Greenberg, S., and Forlines, C., Enabling Interaction with Single User Applications through Speech and Gestures on a Multi-User Tabletop, in Proceedings of AVI'06 (Venezia, Italy, May 2006), ACM Press, 336-343.

14. Van der Veer, G., Welie, M., Task Based Groupware Design: Putting Theory into Practice, in Proceedings of DIS'00 (New York NY, August 2000), ACM Press, 326-337.

15. Van der Veer, G., Welie, M, Chisalita, C., Introduction to Groupware Task Analysis, in Proceedings of TAMODIA'02 (Bucarest, Romania, July 2002), ACM SIGCHI, 32-39.

16. Vernier, F., Nigay, L., A Framework for the Combination and Characterization of Output Modalities, in Proceedings of DSV-IS'00 (Limerick, Ireland, June 2000), Springer, 32-48. 